\title{
Neuroprotective mechanism of HIF-1 $\alpha$ overexpression in the early stage of acute cerebral infarction in rats
}

\author{
YUHUA SUN, WEIYA HE and LIJIAO GENG
}

Department of Neurology, Henan University Huaihe Hospital, Kaifeng, Henan 475001, P.R. China

Received February 2, 2015; Accepted March 17, 2016

DOI: 10.3892/etm.2016.3288

\begin{abstract}
The present study aimed to explore the expression and neuroprotective mechanism of hypoxia inducible factor $(\mathrm{HIF}-1 \alpha)$ in the brain tissue of a rat model of early acute cerebral infarction. A total of 64 Sprague Dawley rats were randomly divided into surgery and sham groups and the model of focal cerebral infarction was established by the suture-occluded method. In the sham group, blood vessels were separated but not occluded. Rats in the surgery and sham groups were subdivided into eight groups ( $\mathrm{n}=4 /$ group). Blood samples was collected at 8 time points including $30 \mathrm{~min}$ and $1,3,6,12,48,24$ and $72 \mathrm{~h}$, respectively, and HIF- $1 \alpha$ content was detected using ELISA. Brain tissues of rats in all groups were harvested following blood collection. HIF-1 $\alpha$ protein expression was detected by immunohistochemistry and terminal deoxynucleotidyl transferase (TdT) dUTP nick-end labeling was used to analyze the brain cell apoptosis index. ELISA results demonstrated that rats in the surgery group began to express HIF-1 $\alpha$ within 30 min, and HIF-1 $\alpha$ expression levels gradually increased, peaking at $12 \mathrm{~h}$. HIF-1 $\alpha$ expression levels were significantly increased in the surgery group at all time points, as compared with the sham group $(\mathrm{P}<0.05)$. The concentration of HIF-1 $\alpha$ decreased rapidly in $12 \mathrm{~h}$. At various time points, HIF-1 $\alpha$ protein expression in the brain tissue of rats in the sham group was negative. HIF-1 $\alpha$ protein expression was significantly increased in the surgery group $(\mathrm{P}<0.05)$, peaking at $12 \mathrm{~h}$, and decreasing after this point. As compared with the sham group, the apoptosis indices of the brain tissue of rats in the surgery group exhibited a gradual increasing trend with significant decreases observed after $12 \mathrm{~h}(\mathrm{P}<0.05)$. Intra-group comparison of all indices in the surgery group, indicated that there was a statistically significant difference between postoperative $12 \mathrm{~h}$ and other time points $(\mathrm{P}<0.05)$. In conclusion, the present study demonstrated that HIF-1 $\alpha$ was highly expressed in the brain tissue of rat models of
\end{abstract}

Correspondence to: Dr Weiya He, Department of Neurology, Henan University Huaihe Hospital, 115 Ximen Street, Kaifeng, Henan 475001, P.R. China

E-mail: weiyahe@yeah.net

Key words: acute cerebral infarction, hypoxia inducible factor-1 $\alpha$, cell apoptosis early acute cerebral infarction. The results also indicated that HIF-1 $\alpha$ significantly reduced the apoptosis of infarcted cells, suggesting that HIF-1 $\alpha$ may have a neuroprotective role in early acute cerebral infarction.

\section{Introduction}

As society has developed in China, the average age of the population has increased considerably. Consequently, the incidence of cerebrovascular disease has increased, and appears to be affecting younger individuals (1). Epidemiological survey results in recent years have demonstrated that cerebrovascular disease accounts for the second highest mortality rates in China, second only to malignant tumors (2). As for the survivors, $\sim 75 \%$ of patients with cerebrovascular disease lose the capacity to work, to varying degrees and $>40 \%$ may be severely disabled $(3,4)$. Therefore, cerebrovascular disease is associated with high morbidity and mortality, causing serious harm to human health and quality of life. Cerebral infarction accounts for $>70 \%$ of all cerebral vascular diseases, therefore research on cerebral infarction is urgently required (5). Acute cerebral infarction (ACI) refers to disturbance in the blood supply to brain tissue due to stenosis or occlusion of the intracranial or extracranial arteries, which leads to ischemic necrosis or cerebromalacia due to focal brain tissue ischemia and hypoxia (6). Cells in the central area of severe ischemia do not survive severe ischemia and hypoxia, which is the irreversible process $(7,8)$. Previous studies have demonstrated that regional blood flow in the area surrounding the cerebral infarction, which is named the penumbra, reduced and exhibited a low perfusion state $(5,9)$. Therefore, the brain cells were apoptotic and if they could be protected in a timely manner, the occurrence of apoptosis may be reduced and the process of cell death may be simultaneously delayed. Nerve cell apoptosis in the ischemic penumbra has been demonstrated to be an important type of cell death during ischemic cerebrovascular disease (10). Various stress factors, including HIF-1 $\alpha$, are produced during apoptosis. These stress factors have been demonstrated to combine with their corresponding target gene to regulate the molecular pathways of hypoxia-responsive genes, which is an important mechanism for the maintenance of mammalian homeostasis under hypoxic conditions $(11,12)$. The HIF-1 $\alpha$ signal transduction pathway may improve blood supply to cerebral tissue and reduce hypoxia-induced ischemic injury. Therefore, HIF-1 $\alpha$ may be a novel molecular target for 
neuroprotective therapy following ischemic brain injury, and may suggest a novel direction for the research of neuroprotective drugs. Previous studies have demonstrated the credibility and clinical significance of this hypothesis $(13,14)$ In the present study, rat models of ACI were established in order to observe the expression of HIF-1 $\alpha$ in the brain tissue of rats during the early stage of ACI, and to investigate the underlying mechanism.

\section{Materials and methods}

Model preparation. A total of 64 male Sprague Dawley rats, weighing 200-220 g and aged 2 months, were purchased from Henan Provincial Experimental Animal Center (Zhengzhou, China). All rats were maintained in a temperature $\left(22 \pm 1^{\circ} \mathrm{C}\right)$ and humidity $(60 \%)$ controlled environment, with a $12 \mathrm{~h}$ light/dark cycle and ad libitum access to food and water. Rat models of middle cerebral artery infarction were established according to the suture-occluded method. Rats were fasted for $12 \mathrm{~h}$ prior to the surgery. Rats were anesthetized with $0.3 \mathrm{ml} / 100 \mathrm{~g}$ chloral hydrate (10\%; Sangon Biotech Co., Ltd., Shanghai, China) via intraperitoneal injection. Briefly, an incision was made along the midline of the neck and the external carotid arteries were double ligated (5/0 ligature; Beijing Dingguo Biotechnology Co., Ltd., Beijing, China) and amputated $\sim 3 \mathrm{~mm}$ from the bifurcation of the common carotid artery. A small incision was made in the common carotid artery. The head end of a fishing line was burned into a sphere (diameter, $\sim 0.205 \mathrm{~mm}$ ), and slowly inserted into the incision to $18-20 \mathrm{~mm}$, until resistance was detected. The clamp on the arteries was simultaneously released and the fishing line and common carotid artery were ligated together and sutured. Following this procedure, the rats exhibited listlessness, ipsolateral Horner's disease, contralateral forelimb droop, adduction, internal rotation and spontaneously circling to the affected side $2 \mathrm{~h}$ after the surgery. These symptoms indicated that modeling was successful $(n=32)$ and rats without these symptoms were eliminated from the present study. The blood vessels of rats in the sham group were separated but not occluded. Rats in the surgery and sham groups were subdivided into eight groups, with four rats in each group, according to the time point at which blood samples were collected (30 min and 1, 3, 6, 12, 48, 24 and $72 \mathrm{~h}$, respectively). The present study was performed in strict accordance with the recommendations outlined in the Guide for the Care and Use of Laboratory Animals of the National Institutes of Health (15). The protocol for the present study was reviewed and approved by the Institutional Animal Care and Use Committee of Henan University Huaihe Hospital (Kaifeng, China).

ELISA. Blood samples were collected from rats in the surgery and sham groups at eight time points: $30 \mathrm{~min}$ and 1, 3, 6, 12, 48, 24 and $72 \mathrm{~h}$, respectively. After standing at room temperature for $1 \mathrm{~h}$, the whole blood was centrifuged at 3,000 x $\mathrm{g}$ for $15 \mathrm{~min}$ and the liquid supernatant was extracted for ELISA detection. ELISA was performed according to the protocol outlined in the HIF-1 $\alpha$ ELISA kit (Bioco Laibo Technology Co., Ltd, Beijing, China) to assess the levels of HIF-1 $\alpha$ in both groups at the various time points. Three holes were used for each sample and standard. Optical density values were measured at
$492 \mathrm{~nm}$ using a microplate reader (Miltiskan MK3; Thermo Fisher Scientific, Inc., Waltham, MA, USA).

Immunohistochemistry. Brain tissues in all groups were perfused with $4 \%$ paraformaldehyde (Sangon Biotech Co., Ltd.) following blood collection. Specimens were continuously cut into $0.3 \mu \mathrm{m}$ sections and embedded by paraffin. The samples were adhered to the glass slides, treated by polylysine (Sangon Biotech Co., Ltd.) and incubated at $60^{\circ} \mathrm{C}$ for $1 \mathrm{~h}$ prior to treatment with xylene and 100, 95, 80 and 75\% ethanol, successively. Subsequently, the samples were washed three times with distilled water and immersed in $3 \% \mathrm{H}_{2} \mathrm{O}_{2}$ for $10 \mathrm{~min}$ to remove the endogenous hydrogen peroxide enzyme. Following washing three times with phosphate-buffered saline with Tween 20 (PBST; Sangon Biotech Co., Ltd.) for $5 \mathrm{~min}$, citric acid buffer (Sangon Biotech Co., Ltd.) was added and the sample was microwaved at moderate heat for $3 \mathrm{~min}$, and subsequently cooled. This procedure was repeated twice for antigen retrieval. Samples were washed three times with PBST for $5 \mathrm{~min}$ and incubated with $5 \%$ bovine serum albumin at room temperature for $30 \mathrm{~min}$. Following washing three times with PBST for $5 \mathrm{~min}$, the samples were incubated with HIF-1 $\alpha$ monoclonal antibody (1:100; cat. no. BM0912; Boster Biotechnology Co., Wuhan, China) at $4^{\circ} \mathrm{C}$ overnight. The samples were washed three times with PBST for $5 \mathrm{~min}$ and incubated with HRP-conjugated goat anti-mouse secondary antibody (1:500; cat. no. CSB-SA11952m; Qianjian Biotechnology Co., Shanghai, China) at room temperature for $1 \mathrm{~h}$. Subsequently, the samples were washed three times with PBST for $5 \mathrm{~min}$, stained with 3,3'-diaminobenzidine (Sangon Biotech Co., Ltd.), followed by staining with hematoxylin (Boster Biotechnology Co.), differentiated with $0.1 \%$ hydrochloric acid, and retuned to blue with PBS. Routine dehydration and transparency using a gradient method were conducted. Neutral resin (Boster Biotechnology Co.) was used for sealing. Cells were deemed to be positively stained if their nuclei were brown/yellow and part of cytoplasm was stained, otherwise the cells were deemed negative. Five fields were randomly selected under high magnification (magnification, x400; AX80; Olympus Corporation, Tokyo, Japan) and 200 nerve cells were assessed for positive staining. The HIF-1 $\alpha$ label index (LI) was calculated as follows: LI=positive staining cell number/total number of nerve cells x $100 \%$, $\mathrm{LI}>5 \%$ was indicative of positive expression of HIF-1 $\alpha$.

Terminal deoxynucleotidyl transferase (TdT) dUTP nick-end labeling (TUNEL). Brain tissue sections from rats in all groups were dewaxed and washed three times with PBST for $5 \mathrm{~min}$. TUNEL was performed according to manufacturer's protocol (Roche Diagnostics GmbH, Basel, Switzerland). A total of 10 fields surrounding the infarction and peripheric regions were randomly selected and analyzed under a microscope (magnification, x400; AX80; Olympus Corporation). The brain cell apoptosis index was calculated as the proportion of apoptotic cells in the total cells.

Statistical analysis. All data were analyzed with SPSS 17.0 statistical software (SPSS, Inc., Chicago, IL, USA). Measurement data were expressed as the mean \pm standard deviation and were compared between multiple groups using analysis of variance. 
Comparison between groups was conducted according to the least significant difference method. $\mathrm{P}<0.05$ was considered to indicate a statistically significant difference.

\section{Results}

Serum HIF-1 $\alpha$ expression increases during ACI, peaking at $12 \mathrm{~h}$. As shown in Fig. 1, serum concentrations of HIF-1 $\alpha$ were significantly higher in the rats of the surgery group, as compared with the sham group $(\mathrm{P}<0.05)$, at all eight of the time points, including $30 \mathrm{~min}$ and 1, 3, 6, 12, 24, 48 and $72 \mathrm{~h}$. Rats in the surgery group exhibited dynamic changes in serum HIF-1 $\alpha$ concentration. HIF-1 $\alpha$ concentration levels began to increase during the $30 \mathrm{~min}$ postoperative period, and peaked at $12 \mathrm{~h}$. Significant differences in the concentration of HIF-1 $\alpha$ were detected between the surgery and sham groups, and among all the time points in the surgery group in $12 \mathrm{~h}(\mathrm{P}<0.05)$. Although serum HIF-1 $\alpha$ concentration gradually decreased in the surgery group between 12 and $72 \mathrm{~h}, \mathrm{HIF}-1 \alpha$ concentration remained significantly higher than the sham group $(\mathrm{P}<0.05)$. In conclusion, serum HIF-1 $\alpha$ expression levels peaked at $12 \mathrm{~h}$ after acute cerebral infarction, and decreased after this point.

HIF-1 $\alpha$ expression in brain tissue increases during ACI, peaking at $12 \mathrm{~h}$. Immunohistochemical analysis demonstrated that HIF-1 $\alpha$ was predominantly expressed in the nuclei of nerve cells, and was also expressed in the cytoplasm. HIF-1 $\alpha$ was successfully detected in the rats of the surgery group (Fig. 2A), whereas no positive expression of HIF-1 $\alpha$ was detected in the rats of the sham group (Fig. 2B). Within the brain tissue of rats in the surgery group, HIF-1 $\alpha$ expression was highest at $12 \mathrm{~h}$ post-surgery, with the proportion of HIF-1 $\alpha$-positive cells gradually decreasing in a time-dependent manner. Significant differences in the proportion of HIF-1 $\alpha$-positive cells were detected between the surgery and sham groups, and among the various time points within the surgery group $(\mathrm{P}<0.05$; Fig. $2 \mathrm{C})$. In conclusion, HIF-1 $\alpha$ expression levels in brain tissue peaked at $12 \mathrm{~h}$ after acute cerebral infarction, and decreased after this point.

During ACI, apoptosis increases in a time-dependent manner, peaking at $12 \mathrm{~h}$. TUNEL staining demonstrated that, as compared with the sham group, volume of apoptotic cells in the surgery group was reduced and they were triangular or fan-shaped (Figs. 3A and B). Furthermore, the Nissl's body was not detectable, nucleus pycnosis was deeply stained and nuclear chromatin was condensed. The chromatin condensed around the nucleus and appeared as crescent, annular or nuclear debris. Apoptosis-negative cell nuclei were not colored. The proportion of apoptotic cells in the brain tissue of rats in the sham group was markedly reduced, as compared with the surgery group, which exhibited obvious cell apoptosis. In the surgery group, the proportion of apoptotic cells increased in a time-dependent manner, peaking at $12 \mathrm{~h}(\mathrm{P}<0.05)$, and gradually decreasing thereafter. Significant differences in the proportion of apoptotic cells were detected between the surgery and sham groups, and among the various time points within the surgery group $(\mathrm{P}<0.05)$ (Fig. $3 \mathrm{C})$. In conclusion, HIF-1 $\alpha$ expression levels in brain tissue peaked at $12 \mathrm{~h}$ after acute cerebral infarction, and decreased after this point.

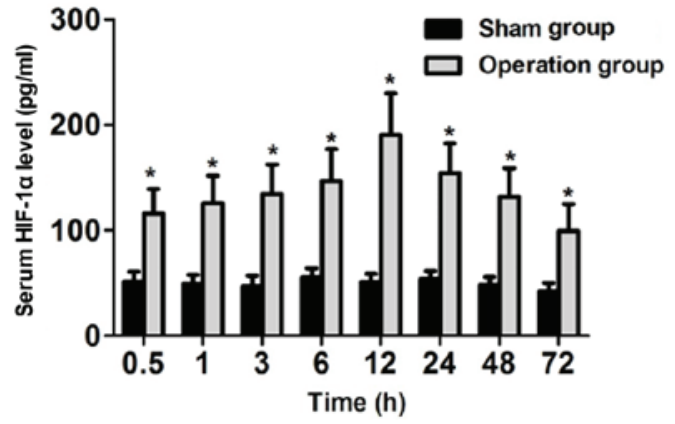

Figure 1. Comparison of serum HIF-1 levels in rats at different time points following surgery. ${ }^{*} \mathrm{P}<0.05$ vs. the sham group.

\section{Discussion}

Patients with acute ischemic cerebrovascular disease suffer from high rates of morbidity and mortality (16). The probability of patients experiencing recurrent stroke within five years is $20 \%$ in patients who have already had a stroke, which seriously affects the quality of life of these patients $(17,18)$. Therefore, investigating cerebral infarction pathogenesis, prevention, timely diagnosis and treatment following disease is likely to significantly reduce the incidence of cerebral infarction and improve the prognosis of patients with ACI. The 'reducible penumbra' and 'therapeutic time window' ACI theories describe a period within 3-6 h of hyperacute cerebral infarction where the focal ischemic brain tissue is in the reversible stage (19). These theories suggest that, if timely arteriovenous thrombolysis was conducted within this period, the ischemic brain tissue may be completely recovered. Nerve cell apoptosis in ischemic penumbra has been demonstrated to be an important cell death mode in patients with ischemic cerebrovascular disease, which also confirmed the existence of a substance (yet to be elucidated) which could alleviate the cell apoptosis in early ACI $(20,21)$.

HIF- $1 \alpha$ is a transcription regulator which has been demonstrated to be widely distributed in mammals under hypoxic conditions (22). Hypoxia is an important pathophysiological factor of cerebral infarction and HIF-1 $\alpha$ has shown to be associated with the response of mammalian cells to ischemic hypoxia (23). The present study demonstrated that HIF-1 $\alpha$ is associated with the pathophysiological process of cerebral infarction, and as such has an influence over cell apoptosis. Under normoxic conditions, HIF-1 $\alpha$ is vulnerable to degradation. This degradation process is blocked under hypoxic conditions, resulting in the accumulation of HIF-1 $\alpha$ (24). Under hypoxic conditions, HIF-1 $\alpha$ is capable of regulating anaerobic metabolism by inducing the expression of its target gene, and promoting angiogenesis and an increase of erythropoietin, which ensures that the hypoxic tissues and cells maintain at a certain oxygen concentration and tolerate the hypoxic environment $(25,26)$. Previous studies have demonstrated that, when cerebral ischemia occurs, HIF-1 $\alpha$-induced gene expression may promote reperfusion of the ischemic penumbra area, thus improving glucose transport, which helps to mediate the ability of cells to tolerate hypoxia and has an important protective role for ischemic and hypoxic neurons $(27,28)$. Following mild transient ischemia and hypoxia, HIF-1 $\alpha$ expression levels 

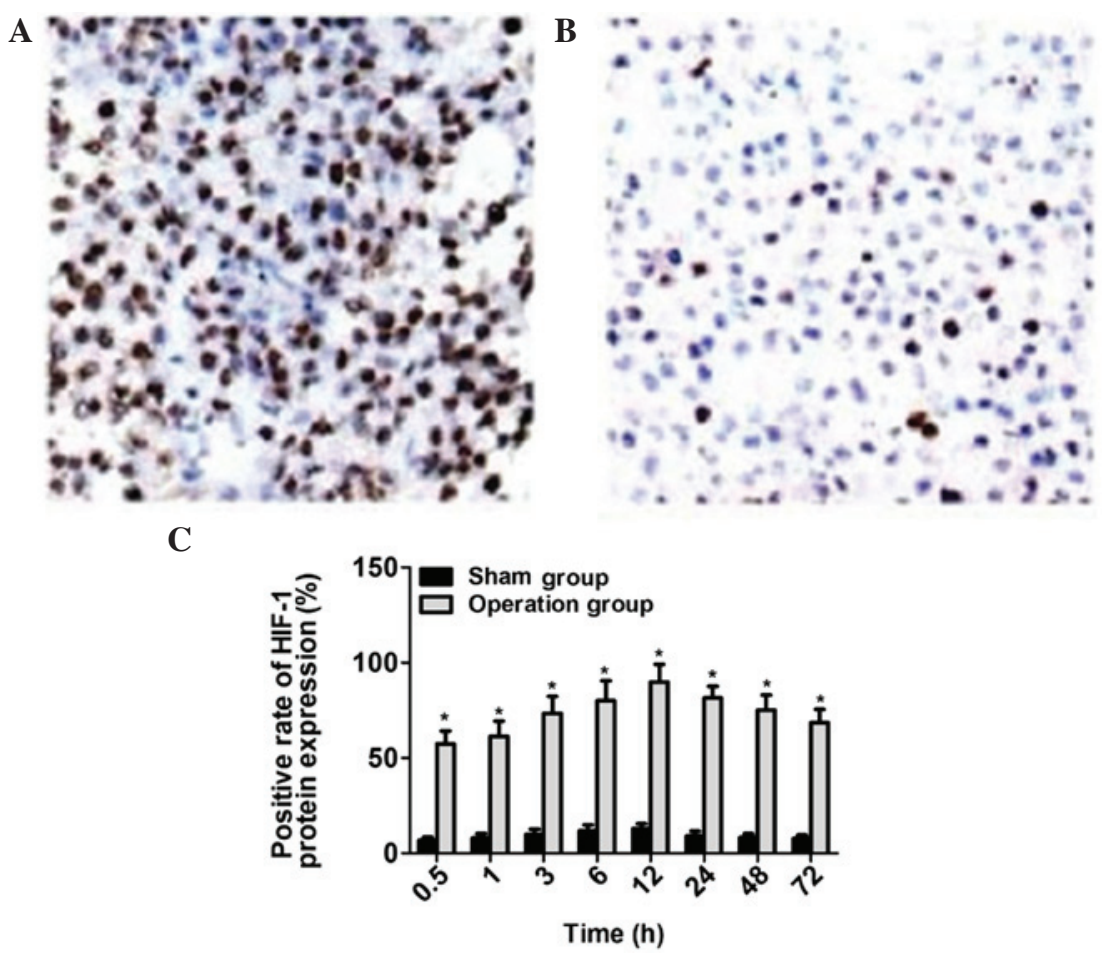

Figure 2. Immunohistochemical analysis of the surgery and sham groups. (A) HIF-1 $\alpha$ was densely expressed in the surgery group, whereas (B) no HIF-1 $\alpha$ expression was detected in the sham group (magnification, $\mathrm{x} 400$ ). (C) Proportion of cells positive for HIF-1 protein expression in the brain tissue of rats in each group at various time points. ${ }^{*} \mathrm{P}<0.05$ vs. the sham group.
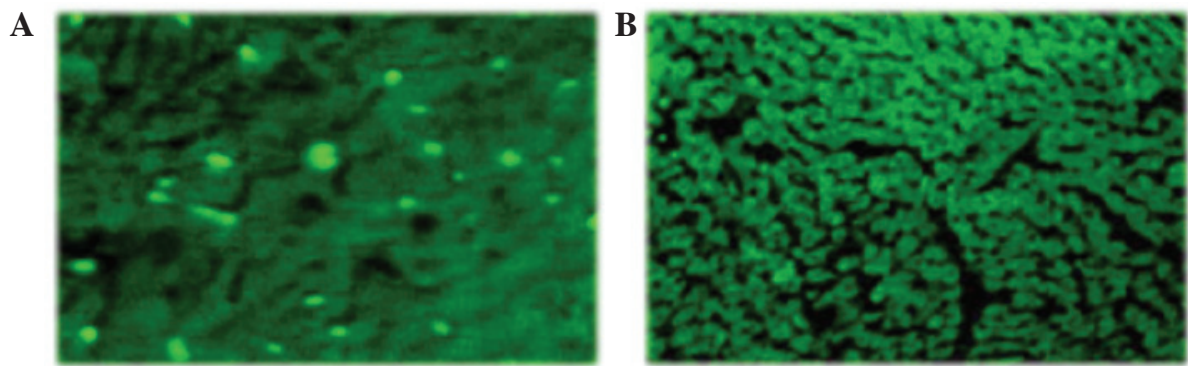

C

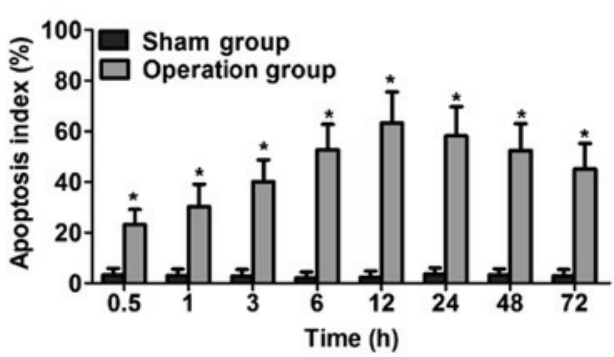

Figure 3. Terminal deoxynucleotidyl transferase (TdT) dUTP nick-end labeling was used for apoptosis index analysis in the (A) surgery group and (B) sham groups (magnification, $\mathrm{x} 400$ ). (C) Quantitative analysis of the apoptosis index at various time points in the surgery and sham groups. " $\mathrm{P}<0.05 \mathrm{vs.}$ the sham group.

are increased, which induces ischemic and hypoxic tolerance and demonstrates the neuroprotective effect of HIF-1 $\alpha$ against ischemia and hypoxia. Chen et al (29) have previously established a rat model of focal cerebral infarction using the modified suture and occlusion of the right middle cerebral artery method. The concentrations of HIF-1 $\alpha$ and VEGF were detected in blood using the ELISA method. The results indicated that HIF-1 $\alpha$ concentration levels began to increase during hyperacute cerebral infarction and peaked within $12 \mathrm{~h}$, which has an important role on a series of hypoxic responses after cerebral infarction. VEGF concentration levels began to increase within $30 \mathrm{~min}$, peaked within $24 \mathrm{~h}$, which indicated that VEGF was also involved in the high expression of cerebral infarction. HIF-1 $\alpha$ and VEGF were found to be significantly correlated.

Notably, the expression of HIF-1 $\alpha$ has been demonstrated to promote nerve cell apoptosis after long-term severe ischemia and hypoxia (30). HIF-1 $\alpha$ induced nerve cell toxicity and 
aggravated ischemic and hypoxic injury of cerebral infarction. Hua et al (31) have previously established a rat model of focal cerebral infarction and observed the effects of recombinant human erythropoietin (RhEPO) on the protein expression of HIF-1 $\alpha$ and neuroprotective effects following cerebral infarction using nerve score, 2,3,5-triphenyltetrazolium chloride staining and immunohistochemistry. The results demonstrated that RhEPO was capable of reducing HIF-1 $\alpha$ protein expression following cerebral ischemic infarction. Furthermore, the results also indicated that earlier intervention induced a greater reduction in the expression of HIF-1 $\alpha$ and recovered neurological function to a greater degree. Therefore, to intensify the research on HIF-1 $\alpha$ in early ACI is particularly important.

In conclusion, the results of the present study indicate that serum HIF-1 $\alpha$ expression levels in brain tissue peak at $12 \mathrm{~h}$ after acute cerebral infarction, and decreased after this time point. This may be a result of the up-regulation of HIF-1 $\alpha$ to protect hypoxia ischemic brain tissue. High expression levels of HIF-1 $\alpha$ regulate cytokines expressing VEGF in areas of hypoxia ischemia, promote the formation of new blood vessels, and, therefore, serve a role in the protection of brain tissue in early acute cerebral infarction.

\section{References}

1. Wang J, An Z, Li B, Yang L, Tu J, Gu H, Zhan C, Liu B, Su TC and Ning $X$ : Increasing stroke incidence and prevalence of risk factors in a low-income Chinese population. Neurology 84: 374-381, 2015.

2. Zhang $\mathrm{H}$, Ju Z, Wang N, Zhang YF, Xu T and Zhang YH: Pulse pressure and in-hospital mortality and morbidity in acute stroke patients. Zhong Hua Gao Xue Ya Za Zhi 16: 633-636, 2008 (In Chinese).

3. Wang SS, Wang JJ, Wang PX and Chen R: Determinants of fatigue after first-ever ischemic stroke during acute phase. PLoS One 9: e110037, 2014

4. Wira CR III, Rivers E, Martinez-Capolino C, Silver B, Iyer G, Sherwin R and Lewandowski C: Cardiac complications in acute ischemic stroke. West J Emerg Med 12: 414-420, 2011.

5. Li J: Clinical characteristics observed in elderly patients with cerebral infarction. Zhong Guo Shi Yong Shen Jing Ji Bing Za Zhi 14: 59-60, 2011 (In Chinese).

6. Fang H, Song B, Cheng B, Wong KS, Xu YM, Ho SS and Chen XY: Compensatory patterns of collateral flow in stroke patients with unilateral and bilateral carotid stenosis. BMC Neurol 16: 39, 2016.

7. Saqqur M, Ibrahim M, Butcher K, Khan K, Emery D, Manawadu D, Derksen C, Schwindt B and Shuaib A: Transcranial Doppler and cerebral augmentation in acute ischemic stroke. J Neuroimaging 23: 460-465, 2013.

8. Iwata T: Current and future prospects of endovascular treatment for acute ischemic stroke. Rinsho Shinkeigaku 54: 1200-1202, 2014 (In Japanese).

9. Fan CG and Guo S: Effect of Ginkgo biloba extract on acute cerebral infarction rats apoptosis genes. Zhong Guo Yi Yuan Yao Xue Za Zhi 35: 902-907, 2015 (In Chinese)

10. Teasell R, Rice D, Richardson M, Campbell N, Madady M, Hussein N, Murie-Fernandez M and Page S: The next revolution in stroke care. Expert Rev Neurother 14: 1307-1314, 2014.

11. Li L, Candelario KM, Thomas K, Wang R, Wright K, Messier A and Cunningham LA: Hypoxia inducible factor-1 $\alpha$ $(\mathrm{HIF}-1 \alpha)$ is required for neural stem cell maintenance and vascular stability in the adult mouse SVZ. J Neurosci 34: 16713-16719, 2014
12. Chen L, Pei H, Zhu S, Zhu J and Shi R: Expression and significance of hypoxia-inducible factor- $1 \alpha$ in lung tissues of obesity-asthma rat. Xi Bao Yu Fen Zi Mian Yi Xue Za Zhi 30: 1262-1265, 2014 (In Chinese).

13. Salva E, Turan SO, Eren F and Akbuğa J: The enhancement of gene silencing efficiency with chitosan-coated liposome formulations of siRNAs targeting HIF-1 $\alpha$ and VEGF. Int J Pharm 478: 147-154, 2014.

14. Chen C, Ostrowski RP, Zhou C, Tang J and Zhang JH: Suppression of hypoxia-inducible factor-1alpha and its downstream genes reduces acute hyperglycemia-enhanced hemorrhagic transformation in a rat model of cerebral ischemia. J Neurosci Res 88: 2046-2055, 2010.

15. Institute of Laboratory Animal Resources (US). Committee on Care, Use of Laboratory Animals, and National Institutes of Health (US). Division of Research Resources: Guide for the care and use of laboratory animals. 8th edition. National Academies Press, Washington, DC, 2011.

16. Walcott BP, Boehm KM, Stapleton CJ, Mehta BP, Nahed BV and Ogilvy CS: Retrievable stent thrombectomy in the treatment of acute ischemic stroke: Analysis of a revolutionizing treatment technique. J Clin Neurosci 20: 1346-1349, 2013.

17. Rahme R, Abruzzo TA and Ringer AJ: Acute ischemic stroke in the setting of cervical carotid occlusion: A proposed management strategy. World Neurosurg 76 (Suppl 6): S60-S65, 2011.

18. Amar AP: Brain and vascular imaging of acute stroke. World Neurosurg 76 (Suppl 6): S3-S8, 2011.

19. Zhu Y, Sun Y, Mao XO, Jin KL and Greenberg DA: Expression of poly (C)-binding proteins is differentially regulated by hypoxia and ischemia in cortical neurons. Neuroscience 110: 191-198, 2002.

20. Donohue A, McLaughlin C, Crowe M and Horgan F: Clinical guideline adherence by physiotherapists working in acute stroke care. Ir Med J 107: 287-289, 2014.

21. El-Koussy M, Schroth G, Brekenfeld C and Arnold M: Imaging of acute ischemic stroke. Eur Neurol 72: 309-316, 2014.

22. Bentovim L, Amarilio R and Zelzer E: HIF1 $\alpha$ is a central regulator of collagen hydroxylation and secretion under hypoxia during bone development. Development 139: 4473-4483, 2012.

23. Sharma S, Kapahi R, Sambyal V, Guleria K, Manjari M, Sudan M, Uppal MS and Singh NR: No association of hypoxia inducible factor-1 $\alpha$ gene polymorphisms with breast cancer in north-west indians. Asian Pac J Cancer Prev 15: 9973-9978, 2014.

24. Tennant DA, Frezza C, MacKenzie ED, Nguyen QD, Zheng L, Selak MA, Roberts DL, Dive C, Watson DG, Aboagye EO and Gottlieb E: Reactivating HIF prolyl hydroxylases under hypoxia results in metabolic catastrophe and cell death. Oncogene 28: 4009-4021, 2009.

25. Deng J, Huang Q, Wang Y, Shen P, Guan F, Li J, Huang H and Shi C: Hypoxia-inducible factor-1alpha regulates autophagy to activate hepatic stellate cells. Biochem Biophys Res Commun 454: 328-334, 2014.

26. Botlagunta M: Neuronal pentraxin 1 expression is regulated by hypoxia inducible factor- $1 \alpha$. Biochem Biophys Res Commun 456: 662-665, 2015.

27. Hu Q, Liang X, Chen D, Chen Y, Doycheva D, Tang J, Tang J and Zhang JH: Delayed hyperbaric oxygen therapy promotes neurogenesis through reactive oxygen species/hypoxia-inducible factor- $1 \alpha / \beta$-catenin pathway in middle cerebral artery occlusion rats. Stroke 45: 1807-1814, 2014.

28. Ma Y, Lovekamp-Swan T, Bekele W, Dohi A and Schreihofer DA: Hypoxia-inducible factor and vascular endothelial growth factor are targets of dietary soy during acute stroke in female rats. Endocrinology 154: 1589-1597, 2013.

29. Chen L, Chen SH and Chen JY: Change characteristics and relation study of serum HIF-1, VEGF factor on acute stage cerebral infarction in SD rats. Leshan Shi Fan Xue Yuan Xue Bao 27: 13-18, 2012 (In Chinese)

30. Panchision DM: The role of oxygen in regulating neural stem cells in development and disease. J Cell Physiol 220: 562-568, 2009.

31. Hua W, Zhou M, Liu W and Qi JP: Effect of RhEPO to hypoxia-inducible factor- $1 \alpha$ after cerebral ischemia. Ha Er Bin Yi Ke Da Xue Xue Bao 47: 216-219, 2013 (In Chinese). 